\title{
Optimizing Systemic Therapy for Bladder Cancer
}

\author{
Sumanta K. Pal, MDa; Matthew I. Milowsky, MD ${ }^{\mathrm{b}}$; and Elizabeth R. Plimack, MDc
}

\begin{abstract}
Over the past several decades, few new systemic agents have been incorporated into the treatment paradigm for bladder cancer. Platinum-based therapy remains the cornerstone of treatment in the perioperative and metastatic settings. Despite level one evidence, use of cisplatin-based therapy in the neoadjuvant setting has been dismal. Second-line therapy for metastatic disease has only modest activity with no survival benefit. However, the elucidation and investigation of novel molecular targets, new therapeutics, and associated biomarkers with strong biologic rationale are actively changing the landscape in bladder cancer. Although the field is moving rapidly, no new drug approvals are currently pending and a need remains to continue to educate the medical oncology and urology communities on the optimal use of currently available treatments. This article outlines the evidence, including that from prospective studies and meta-analyses, providing the basis for the current recommendations from NCCN, and details previous and ongoing studies of targeted therapy for bladder cancer. (JNCCN 2013;11:793-804)
\end{abstract}

Over the past decade, several advances in systemic therapy for genitourinary cancers have occurred. Since 2005, a total of 7 agents have been approved for metastatic renal cell carcinoma (RCC) based on positive phase III trials. ${ }^{1}$ Similarly, 4 agents have been approved

\footnotetext{
From the a Department of Medical Oncology \& Experimental Therapeutics, City of Hope Comprehensive Cancer Center, Duarte, California; ${ }^{b}$ Division of Hematology and Oncology, University of North Carolina Lineberger Comprehensive Cancer Center, Chapel Hill, North Carolina; and 'Department of Medical Oncology, Fox Chase Cancer Center, Philadelphia, Pennsylvania.

Submitted February 11, 2013; accepted for publication April 26, 2013.

The authors have disclosed that they have no financial interests, arrangements, affiliations, or commercial interests with the manufacturers of any products discussed in this article or thei competitors. This work was supported by NIH K12 2K12CA00172716A1 (Dr. Pal).

Correspondence: Sumanta K. Pal, MD, Department of Medical Oncology \& Experimental Therapeutics, City of Hope Comprehensive Cancer Center, 1500 East Duarte Road, Duarte, CA 91010.

E-mail: spal@coh.org
}

for metastatic castration-resistant prostate cancer (mCRPC) since 2009.2 This rapid progress has not been observed in bladder cancer. Cisplatin, which remains the cornerstone of systemic therapy for the disease, was first noted to have activity in bladder cancer in $1976 .{ }^{3}$

Furthermore, in metastatic RCC and mCRPC, therapies have evolved that reflect the biology of the disease. For instance, with the knowledge that vascular endothelial growth factor (VEGF) and the mammalian target of rapamycin (mTOR) are key drivers of RCC pathogenesis, novel agents have been developed that successfully target these pathways. ${ }^{1}$ In mCRPC, therapies have been developed to more effectively abrogate androgen receptor-mediated signaling. ${ }^{4}$ In contrast, the systemic management of bladder cancer remains confined to cytotoxic regimens that do not selectively target malignant cells.

Although a lack of novel agents for bladder cancer may exist, no lack of debate exists regarding the therapeutic approach to the disease. The utility of adjuvant chemotherapy after cystectomy remains highly contentious. Neoadjuvant therapy is also the subject of much discussion. Despite the existence of positive phase III data supporting this approach, it appears to be infrequently used. ${ }^{5,6}$ Finally, in the metastatic setting, little consensus exists regarding how to treat patients with refractory disease. This article provides an overview of the data supporting current recommendations from NCCN related to systemic treatments for bladder cancer. ${ }^{7}$ Because of the already broad scope of this review, combined modality treatment (ie, chemoradiation) is not discussed. 


\section{Neoadjuvant Chemotherapy}

For nonmetastatic, muscle-invasive bladder cancer (MIBC) staged as cT2-T4a with no nodal involvement, the NCCN Bladder Cancer Panel has conferred a category 1 (ie, high-level evidence and uniform consensus) recommendation for both radical cystectomy and a strong consideration of cisplatin-based combination therapy. ${ }^{7}$ Chemotherapy, transurethral resection of bladder tumor, or chemoradiation alone is reserved for patients who have extensive comorbidity or poor performance status.

The support for neoadjuvant chemotherapy before cystectomy for MIBC is derived from both prospective, phase III trials (Table 1) and meta-analytic data. The most recent NCCN Clinical Practice Guidelines in Oncology (NCCN Guidelines) include 3 recommended regimens for neoadjuvant, and by extrapolation, adjuvant chemotherapy: cisplatin, methotrexate, and vinblastine (CMV); dosedense methotrexate, vinblastine, doxorubicin, and cisplatin (DD-MVAC); and gemcitabine and cisplatin (GC). ${ }^{7}$ All regimens are a category $2 \mathrm{~A}$ recommendation (lower-level of evidence and uniform consensus). CMV is listed as an option for neoadjuvant therapy, citing recently reported long-term results from the BA06 30894 trial. ${ }^{6}$ The study represents the largest prospective assessment of neoadjuvant therapy for MIBC to date, and randomized 976 patients to receive either definitive therapy alone (ie, cystectomy and/or radiation) or 3 cycles of CMV followed by definitive therapy. ${ }^{8} \mathrm{~A}$ second neoadjuvant regimen for MIBC supported by the NCCN Guidelines is DD-MVAC. ${ }^{7}$ The activity of neoadjuvant methotrexate, vinblastine, doxorubicin, and cisplatin (MVAC) in MIBC was reportedly as early as 1988 , but the randomized phase III SWOG-8710/INT-0080 study assessing the regimen was not published until 2003.5,9 In this study, 317 patients were randomized to receive either MVAC followed by cystectomy or cystectomy alone. Neoadjuvant MVAC was associated with an improvement in overall survival (OS) of 2.6 years (77 vs 46 months; $P=.05$ ). The pathologic complete response (pT0) rate was $38 \%$ for patients treated with chemotherapy versus $15 \%$ for those undergoing cystectomy alone, with those experiencing a pTO achieving an 85\% 5-year survival. Although MVAC was administered over the course of 4 weeks in the SWOG trial, a randomized study in the metastatic setting suggested that a higher response rate could be achieved using a less toxic accelerated regimen of DD-MVAC, in which the same doses of cisplatin and doxorubicin are given on day 1 and 2 every 2 weeks with growth factor support. ${ }^{10}$ Although this dose-dense regimen has not been assessed in a randomized fashion in the neoadjuvant setting, retrospective series suggest comparable efficacy, with Blick et $\mathrm{al}^{11}$ showing a pTO rate of $43 \%$ in a retrospectively assessed sequential cohort of 60 patients treated with DD-MVAC who subsequently underwent surgery.

GC is also an option in the perioperative setting. ${ }^{7}$ Although no formal comparison of GC to MVAC has occurred in the neoadjuvant setting, data from the metastatic setting suggesting equivalence (discussed later) are often used to support this option. ${ }^{12}$ Furthermore, multiple retrospective studies are now available documenting the activity of GC in this setting. ${ }^{13-19}$ The accounts of efficacy in these reports are highly variable; for instance, in a cohort of 29 patients who received neoadjuvant GC, Weight et $\mathrm{al}^{18}$ reported a pT0 rate of only $7 \%$ and a median disease-free survival (DFS) of 10.5 months. In contrast, Dash et $\mathrm{a}^{14}$ reported a pT0 rate of $26 \%$, and a median DFS was not reached with median follow-up

\section{Table 1 Randomized Clinical Trials of Neoadjuvant Chemotherapy That Support Use of This} Modality in Muscle-Invasive Bladder Cancer

\begin{tabular}{|llll|}
\hline Study & N & Randomization & Result \\
\hline $\begin{array}{l}\text { SWOG-8710 } \\
\text { (INT-0800) }\end{array}$ & 317 & $\begin{array}{l}\text { MVAC } \rightarrow \text { surgery vs } \\
\text { surgery alone }\end{array}$ & $\begin{array}{l}\text { Improved overall survival with MVAC (77 vs 46 mo; } \\
P=.05) \\
\text { Improved rate of pT0 disease with MVAC (38\% vs 15\%; } \\
P<.001)\end{array}$ \\
BA06 30894 & 976 & $\begin{array}{l}\text { CMV } \rightarrow \text { surgery/radiation } \\
\text { vs surgery/radiation alone }\end{array}$ & $\begin{array}{l}\text { Improvement in 10-year survival with CMV (36\% vs } \\
30 \%), \text { translating to a 16\% reduction in risk of death }\end{array}$ \\
\hline
\end{tabular}

Abbreviations: CMV, cisplatin, methotrexate, and vinblastine; MVAC, methotrexate, vinblastine, doxorubicin, and cisplatin; pT0, pathologic complete response. 
of 24.2 months. In a systematic review of the relevant literature, Yuh et $\mathrm{al}^{20}$ summarized outcomes across several of these retrospective experiences, including a total of 164 patients in their analysis. The cumulative pTO rate was $25.6 \%$, and $65 \%$ of patients achieved disease that was less than pT2. These response rates support the use of GC in the neoadjuvant setting. Consistently across these reports, downstaging with neoadjuvant chemotherapy (ie, findings of $<$ pT2 disease at the time of cystectomy) was associated with improved DFS.

To address the varied results from prospective assessments, meta-analytic data have been generated to provide a perspective on the efficacy of neoadjuvant cisplatin-based chemotherapy. An updated effort by the Advanced Bladder Cancer (ABC) MetaAnalysis Collaboration, published in 2005, included data from 3005 patients treated across 11 randomized trials. ${ }^{21}$ These pooled data suggest an increase in 5 -year OS and DFS rates of 5\% and 9\%, respectively. A slightly smaller meta-analysis reported by Winquist et $\mathrm{al}^{22}$ included 2605 patients, and suggested a $6.5 \%$ increase in 5 -year OS. Notably, a mortality rate of $1.1 \%$ in association with cisplatin-based combination therapy was reported.

Despite the accumulating evidence for neoadjuvant therapy, estimates from the National Cancer Database suggest that only $34.5 \%$ of patients receive perioperative chemotherapy, including both neoadjuvant and adjuvant therapy. ${ }^{23}$ Although a certain proportion of patients may be poor candidates for therapy based on comorbidity or impaired renal function (thereby precluding cisplatin-based chemotherapy), these factors alone do not explain the underuse of neoadjuvant treatment. Other reasons frequently cited include perceived risks associated with chemotherapy, discomfort with a delay in definitive surgical intervention, and the interpretation within the urologic community that the $9 \%$ increase in disease-specific survival is of small magnitude. ${ }^{24}$

\section{Adjuvant Chemotherapy}

The NCCN Guidelines confer a category 2B recommendation (lower-level of evidence and consensus) for use of adjuvant chemotherapy in patients who have received prior cystectomy (radical or partial) but did not receive neoadjuvant treatment. ${ }^{7}$ This recommendation reflects the relatively limited pool of evidence to support this modality. Although several large, randomized assessments of adjuvant therapy have been reported, these studies have been marred by poor accrual and other issues. For instance, the Spanish Oncology Genitourinary Group (SOGUG) trial 99/01 randomized 142 postcystectomy patients with either pT3-4 or node-positive disease to a regimen of paclitaxel, gemcitabine, and cisplatin (PGC) or observation..$^{25}$ The study ultimately showed a survival advantage with adjuvant PGC compared with observation (median not reached [NR] vs 26 months; $P<.0009$ ). Unfortunately, the study was terminated early and thus underpowered (target accrual 340), with the results only reported in abstract form. Furthermore, PGC chemotherapy was associated with a substantial incidence of grade 3/4 toxicity.

A second phase III study reported by Cognetii et $\mathrm{al}^{26,27}$ randomized 194 postcystectomy patients with pT2G3 or pT3-4 MIBC (irrespective of nodal involvement) to either immediate GC or GC at the time of recurrence. With a median follow-up of 35 months, immediate chemotherapy resulted in no difference in 5-year OS $(P=.24)$. However, this study was also subject to early termination because of poor accrual (target accrual 610). Two other studies, CALGB 90104 and EORTC 30994, also closed early because of slow accrual.

Because of the stated inadequacies of prospective studies assessing adjuvant therapy, meta-analytic data are often invoked to support this modality. The ABC Meta-Analysis Collaboration has assessed individual patient data derived from 491 patients spanning 6 clinical trials. ${ }^{28}$ Collectively, this analysis identified a $25 \%$ reduction in the risk of death with adjuvant chemotherapy, with particular benefit seen in those patients with pT3-4 or node-positive disease at cystectomy. Svatek et $\mathrm{al}^{29}$ assessed offprotocol use of adjuvant therapy in a retrospective cohort of 3947 patients treated at 11 centers. A total of 936 patients $(23.6 \%)$ received adjuvant therapy. After multivariate analysis, the receipt of adjuvant therapy was independently associated with improved survival (hazard ratio [HR], 0.83; 95\% CI, 0.72\%$0.97 \% ; P=.017)$. Akin to the $A B C$ meta-analysis, higher-risk subgroups (composed mainly of patients with pT3-4 disease or nodal involvement) derived greater benefit from adjuvant therapy in terms of both DFS and OS. 


\section{Chemotherapy for Metastatic Disease}

Both GC and DD-MVAC are category 1 recommendations for the treatment of metastatic urothelial carcinoma. Before the development of these regimens, MVAC represented the standard of care in this setting. A randomized trial published by Logothetis et $\mathrm{al}^{30}$ in 1990 compared MVAC with cisplatin, cyclophosphamide, and doxorubicin (CISCA) in $110 \mathrm{pa}-$ tients with metastatic urothelial carcinoma. Survival was improved with MVAC relative to CISCA (62.6 vs 48.3 weeks). To accelerate this regimen and mitigate the hematologic toxicity, EORTC 30924 compared DD-MVAC (essentially twice-weekly MVAC with growth factor support) with traditional MVAC. ${ }^{31}$ In this study, 263 previously untreated patients were randomized to receive either DD-MVAC or MVAC. With a median follow-up of 7.3 years, the HR for mortality favored DD-MVAC (HR, 0.76). Furthermore, fewer patients receiving DD-MVAC died as a consequence of metastatic disease (64.9\% vs $76.0 \%$ ).

Standard MVAC was also directly compared with GC in a phase III study. ${ }^{12,32}$ In this trial, 405 patients with metastatic urothelial cancer were randomized to receive either regimen. Median OS was similar with MVAC and GC (15.2 and 14.0 months, respectively; $P=.66)$. Given the similar efficacy and its lesser toxicity, GC remains preferred over conventional MVAC.

Efforts have been made to build on the backbone of GC chemotherapy with other cytotoxics. A randomized phase II study suggested enhanced response rate and progression-free survival (PFS) with PGC compared with GC. ${ }^{33}$ The same comparison was made in a larger study in EORTC 30987, in which 626 patients with treatment-naïve metastatic urothelial cancer were randomized to receive either GC or PGC. ${ }^{34}$ With a median follow-up of 4.6 years, OS was numerically superior with PGC, although this difference was not statistically significant in the intent-to-treat population (15.8 vs 12.7 months; $P=.075$; the study was powered to detect a 4 -month improvement in OS). However, in the eligible study population, a 3.2-month improvement in OS was observed with PGC (HR, 0.82; $P=.03)$. Despite this improvement, the incidence of thrombocytopenia, bleeding, and febrile neutropenia were all greater with the 3-drug combination. Therefore, the NCCN Guidelines do not advocate the use of PGC for metastatic disease.
Beyond first-line therapy for metastatic bladder cancer, little consensus exists regarding the optimal therapeutic strategy. To date, only the phase III assessment of vinflunine has yielded a survival advantage in this setting. ${ }^{35}$ In this study, 376 patients with metastatic bladder cancer who had progressed after platinum-based therapy were randomized to receive either vinflunine with best supportive care (BSC) or BSC alone. In the eligible population, a significant improvement in OS was seen with vinflunine (6.9 vs 4.3 months; $P=.04)$. However, in the intent-totreat population, although $\mathrm{OS}$ favored vinflunine, the difference was not statistically significant (6.9 vs 4.6 months; $P=.287$ ). Vinflunine is not approved by the FDA for advanced bladder cancer. ${ }^{36}$ Therefore, the NCCN Guidelines suggest potential second-line regimens that are largely supported by phase II data. Taxanes have shown activity in the refractory setting, and gemcitabine (if not used upfront) may also be considered. ${ }^{37-40}$ Single-agent pemetrexed has been assessed in refractory patients with response rates in excess of $25 \%$; studies combining pemetrexed with gemcitabine in a similar setting show only slightly higher activity, counterbalanced by a greater degree of myelosuppression. ${ }^{41-43}$ Other potentially active agents in the refractory setting include ifosfamide, 5-fluorouracil, and methotrexate. ${ }^{44-47}$

Notably, several prognostic schema have been devised for use in the metastatic setting. In the firstline setting, a combination of 4 variables (visceral metastasis, albumin, performance status, and hemoglobin) was initially identified to predict survival in a series of cisplatin-eligible patients at Memorial Sloan-Kettering Cancer Center. ${ }^{48}$ This prognostic model was then externally validated in a series of 308 patients treated on 7 independent protocols for metastatic urothelial cancer. In the second-line setting, a prognostic model, including performance status, hemoglobin, liver metastasis, and time from prior chemotherapy, has been externally validated. ${ }^{49}$

\section{Systemic Therapy for Cisplatin-Ineligible Patients}

In the perioperative setting, the NCCN Guidelines state that carboplatin should not be substituted for cisplatin, because prospective studies and meta-analytic data in the metastatic setting suggest that carboplatin-based therapy is inferior to cispla- 
tin-based therapy, and insufficient prospective data exist to support carboplatin-based regimens as perioperative treatment. ${ }^{7,50-53}$ For patients with borderline creatinine clearance, alternative dosing strategies for cisplatin can be attempted (ie, split dosing over 2 days), with the caveat that these strategies have not been formally compared with traditional dosing. A key challenge for the practitioner is identifying patients as ineligible for cisplatin-based therapy. To this end, expert panels have convened and a consensus definition has emerged. ${ }^{54}$ Table 2 presents a set of clinical characteristics that may serve as eligibility criteria in prospective studies of patients unfit for cisplatin; on a practical level, these same criteria can be applied off-protocol to define this population. Poor performance status, impaired cardiac function, and the presence of certain comorbidities (ie, baseline neuropathy or hearing loss) may identify patients unfit for cisplatin. Furthermore, patients with impaired creatinine clearance (typically $<60 \mathrm{~mL}$ / min) are also poor candidates for cisplatin. However, accurately determining creatinine clearance, particularly in patients older than 65 years, has been challenging; recent studies have shown discordance among the most common formulas used to estimate creatinine clearance, and an overall trend for these formulas to underestimate true measured creatinine clearance. Analysis of a series of 208 patients with urothelial carcinoma treated with cisplatin-based chemotherapy found a poor concordance between calculated creatinine clearance using a variety of formulas and measured creatinine clearance. ${ }^{55}$ No significant association was seen between calculated clearance of less than $60 \mathrm{~mL} / \mathrm{min}$ versus $60 \mathrm{~mL} / \mathrm{min}$ or greater and the safe receipt of 3 or more cycles of cisplatin-based chemotherapy. The authors recommend obtaining a 24-hour urine creatinine clearance before deeming a patient ineligible for cisplatin. ${ }^{55}$

Several options may be considered in patients unfit for cisplatin. The activity of carboplatin and gemcitabine has been evaluated extensively in this setting. ${ }^{56,57}$ In a phase II study conducted by the Hellenic Oncology Group, 56 patients who had an ECOG performance status of 2, creatinine clearance less than $50 \mathrm{~mL} / \mathrm{min}$, or age older than 75 years received this regimen in the first-line metastatic setting. PFS and OS were 4.8 and 7.2 months, respectively. Myelosuppression represented the most frequent toxicity, and 2 treatment-related deaths occurred. Building on the regimen of carboplatin and gemcitabine, another phase II study further assessed the combination of carboplatin and gemcitabine with docetaxel in a mixed population of patients who were either unfit for cisplatin or had MVAC-refractory disease..$^{58}$ Although the PFS and OS achieved in the study were promising (5.0 and 13.1 months, respectively), patients incurred very high rates of hematologic toxicity with this 3-drug regimen. The phase II/III EORTC 30986 study compared carboplatin and gemcitabine with methotrexate, carboplatin, and vinblastine (M-CAVI) in 238 patients who were ineligible for cisplatin based on an ECOG performance status of 2 or a creatinine clearance less than $60 \mathrm{~mL} / \mathrm{min} .{ }^{59}$ Little difference in efficacy was seen between the regimens, with a median OS of 9.3 months with carboplatin and gemcitabine and 8.1 months with M-CAVI $(P=.64)$. However, the incidence of severe toxicity was high-

\begin{tabular}{|c|c|}
\hline Criterion & Specifications \\
\hline Performance status & $\begin{array}{l}\text { ECOG } 2 \\
\text { Karnofsy Performance Status } 60 \%-70 \%\end{array}$ \\
\hline $\mathrm{CrCl}$ & $\begin{array}{l}<1 \mathrm{~mL} / \mathrm{s}(<60 \mathrm{~mL} / \mathrm{min}) \\
\text { Calculated values are likely to underestimate true } \mathrm{CrCl} \\
\text { Suggest using measured } 24 \text {-hour urine } \mathrm{CrCl}\end{array}$ \\
\hline Preexisting toxicity & $\begin{array}{l}\text { Audiometric hearing loss, grade } \geq 2^{\mathrm{b}} \\
\text { Peripheral neuropathy, grade } \geq 2^{\mathrm{b}}\end{array}$ \\
\hline Cardiovascular status & NYHA class III heart failure \\
\hline
\end{tabular}

Abbreviations: $\mathrm{CrCl}$, creatinine clearance; NYHA, New York Heart Association.

aproposed by Galsky et al. ${ }^{54}$ An expert panel has recommended that these criteria may be used in forthcoming trials to define a cisplatin-ineligible study population.

${ }^{b}$ According to Common Terminology Criteria for Adverse Events, Version 4.0. Available at http://ctep.cancer.gov/protocolDevelopment/electronic applications/ctc.htm. Accessed June 6, 2013. 
Pal et al

er with M-CAVI. This study represented the first level 1 evidence supporting a non-cisplatin-based regimen; carboplatin and gemcitabine may thus be considered a standard of care in patients unfit for cisplatin-based therapy.

For patients with adequate cardiac function but other characteristics precluding cisplatin-based therapy, anthracycline-containing regimens may be considered. Galsky et $\mathrm{a}^{60}$ assessed a regimen of dose-dense doxorubicin and gemcitabine followed by carboplatin and paclitaxel in a series of 25 patients with metastatic bladder cancer. Patients were deemed unfit for cisplatin based on a calculated creatinine clearance of 30 to $60 \mathrm{~mL} / \mathrm{min}$, a baseline serum creatinine greater than $1.5 \mathrm{mg} / \mathrm{dL}$, or prior nephrectomy. Five patients (20\%) experienced a complete response, whereas 9 additional patients $(36 \%)$ experienced a partial response, with a median OS of 15 months. Although several patients developed thromboses, the regimen was otherwise well tolerated. Several non-platinumcontaining regimens have also been examined in patients unfit for cisplatin; these include gemcitabine and vinorelbine; gemcitabine and epirubicin; and gemcitabine and oxaliplatin. ${ }^{61-63}$ The challenge in comparing these datasets are the varied criteria used to characterize patients unfit for cisplatin therapy.

\section{Targeted Therapies}

Several attempts have been made to demonstrate activity of novel targeted strategies in metastatic urothelial cancer (Figure 1). The prognostic role of HER2 has been explored extensively in this disease, and efforts to use HER2-directed therapies in bladder cancer have also been reported. ${ }^{64-67}$ Specifically, Hussain et $\mathrm{al}^{68}$ reported a phase II trial that included patients with metastatic urothelial cancer and no prior systemic therapy for metastatic disease who demonstrated HER2 overexpression by either immunohistochemistry, fluorescence in situ hybridization, or serum HER2 quantity. A regimen of PGC with trastuzumab was administered to a total of 44 patients. Myelosuppression was the most frequently observed toxicity, and nearly one-quarter of enrolled patients developed some degree of cardiac dysfunction. The authors reported encouraging response data, with 31 of 44 patients (70\%) experiencing a response. A median OS of 14.1 months was reported for the cohort.
Aside from HER2, preclinical and translational studies have offered support for targeting epidermal growth factor receptor (EGFR), another member of the ErbB family of transmembrane receptors, in bladder cancer. ${ }^{69,70}$ In SWOG 0031, 31 patients with metastatic bladder cancer were treated with daily gefitinib, a small molecule inhibitor of EGFR. ${ }^{71}$ Only one response was observed, and median PFS was a mere 2 months. In a small series, Pruthi et al ${ }^{72}$ treated 20 patients with MIBC with neoadjuvant erlotinib. Like gefitinib, erlotinib is a small molecule inhibitor of EGFR. Seven patients (35\%) with clinical T2 disease were downstaged to non-MIBC and 5 patients (25\%) had no residual disease. Of course, in the context of this single-arm neoadjuvant study, the impact of erlotinib is challenging to assess, particularly because a pTO rate of $15 \%$ was seen in the cystectomy-alone arm of the SWOG neoadjuvant MVAC study, which included patients with clinical T3 and T4a disease and clinical T2. A third small molecule, lapatinib, antagonizes both EGFR and HER2. In a phase II study involving 59 patients with metastatic bladder cancer who had undergone prior platinum therapy, only 1 experienced a partial response. ${ }^{73}$ However, a total of 18 patients (31\%) experienced stable disease, and clinical benefit (ie, either response or stabilization of disease) was found to be associated with EGFR overexpression. Relatively poor PFS and OS were observed in this study (8.6 and 17.9 weeks, respectively). Nonetheless, a larger phase II/III exploration comparing lapatinib and placebo in advanced bladder cancer is underway; this study will be limited to patients characterized as $2+$ or $3+$ by immunohistochemistry (ClinicalTrials. gov identifier: NCT00949455).

Cetuximab, a monoclonal antibody directed at EGFR, has also been assessed clinically in patients with metastatic bladder cancer who had not received therapy for metastatic disease. In a phase II trial, Grivas et $\mathrm{al}^{74}$ randomized 88 patients in a $1: 2$ fashion to receive either standard GC or GC with cetuximab, and found that PFS was numerically greater in the standard-of-care arm (8.5 vs 7.6 months) and median OS was identical (14 months for both arms). However, this study was criticized for the decision early in the study to reduce the gemcitabine dose by $20 \%$ in the cetuximab arm to mitigate an observed increase in thromboembolic evens with the 3-drug combination, resulting in decreased standard-of-care dose intensity in the experimental arm. 


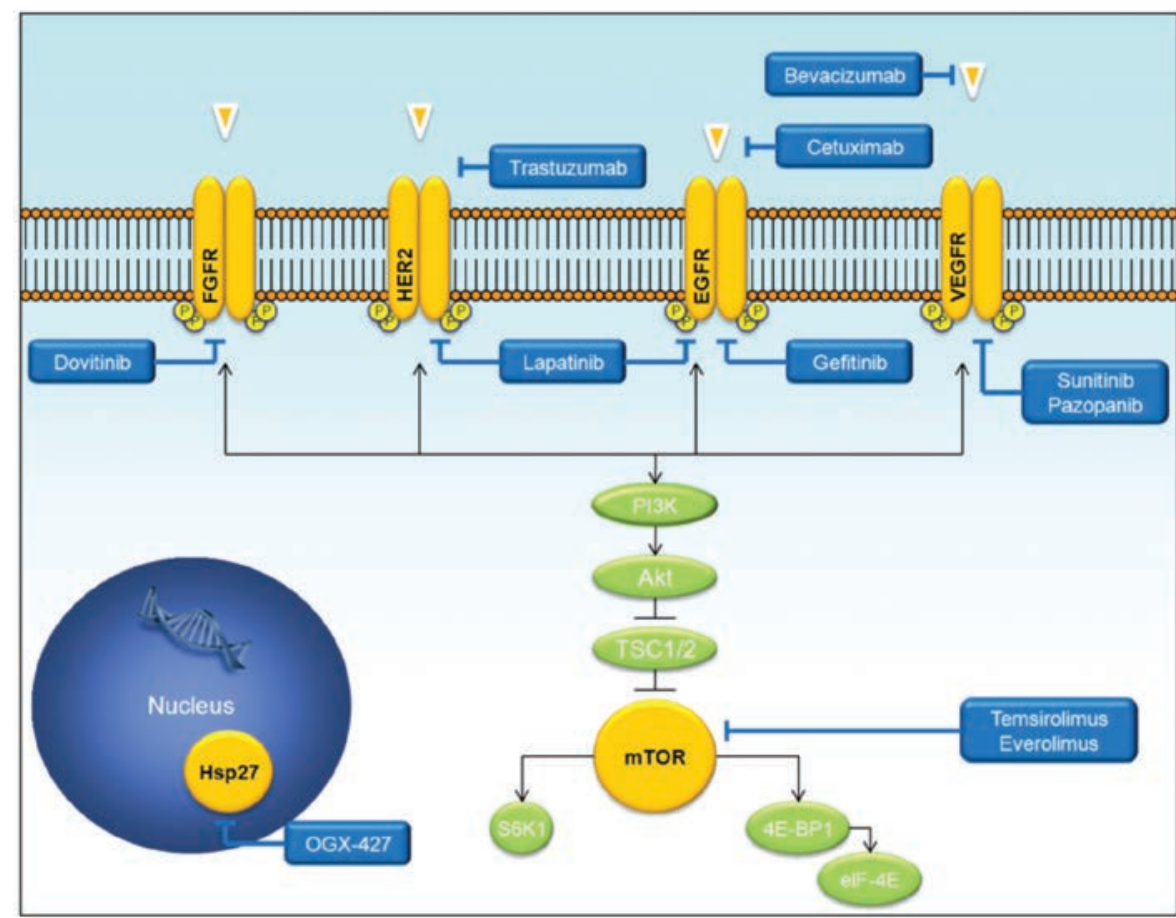

Figure 1 Relevant signaling pathways in bladder cancer. Targeted therapies (depicted in blue boxes) are at varying stages of clinical assessment in patients with bladder cancer.

More encouraging data have emerged from assessment of cetuximab in chemotherapy-refractory patients. In a randomized, noncomparative study, 39 patients with metastatic bladder cancer (who had received 1 prior line of therapy in either the perioperative or metastatic setting) received either cetuximab alone or cetuximab with paclitaxel. ${ }^{75}$ Cetuximab alone showed little efficacy in this setting: 9 of 11 patients showed disease progression within 8 weeks of therapy, leading to early closure of this arm. In contrast, greater clinical benefit was seen with the combination of paclitaxel and cetuximab: 7 of 28 patients $(25 \%)$ showed a response, and median PFS and $O S$ were 16 and 42 weeks, respectively.

In addition to ErbB-mediated signaling, VEGFmediated signaling (and resultant angiogenesis) has been shown in several studies to play a key role in bladder cancer pathogenesis. ${ }^{76}$ Sunitinib, a small molecule with affinity for the VEGF receptor (VEGFR), showed activity in both in vitro and in vivo models of bladder cancer. ${ }^{77}$ As a single agent, sunitinib was assessed in 77 patients with refractory metastatic bladder cancer. ${ }^{78}$ Antitumor activity seemed to be limited, with responses in 4 patients (5\%) and a PFS of 2.3 months overall. Given the noted synergy data with platinum-based therapy, a separate effort was made to combine sunitinib with GC chemotherapy. In 2 parallel phase II studies (one including patients with muscle-invasive disease and the other including patients with metastatic disease), patients were initially treated with standard doses of GC in combination with sunitinib at $37.5 \mathrm{mg}$ daily ( 2 weeks on, 1 week off). ${ }^{79}$ Patients were unable to tolerate full doses of GC in combination with sunitinib, and as a consequence of excess toxicity, both study arms were ultimately closed prematurely. Dismal outcomes were also noted in 2 separate studies of the small molecule VEGFR inhibitor pazopanib in patients with refractory metastatic bladder cancer. $^{80-81}$ Notably, in one of these studies, a discordance was seen between response rates characterized by RECIST criteria and tumor responses characterized by PET and CT densitometry. ${ }^{82}$ As other studies of VEGFR inhibitors have suggested, this may indicate the need for more sophisticated imaging modalities and response criteria to document response to angiogenesis inhibitors. ${ }^{83-85}$

The VEGF-directed monoclonal antibody bevacizumab has also been explored in metastatic bladder cancer. In a phase II study conducted by the Hoosier Oncology Group (HOG), 43 patients with no prior chemotherapy for metastatic disease received 
Pal et al

GC with bevacizumab. ${ }^{86}$ Substantial toxicity was observed, with 3 treatment-related deaths attributable to sudden cardiac death, aortic dissection, and central nervous system hemorrhage, respectively. Other notable toxicities included venous thromboembolism (VTE), with $21 \%$ of patients experiencing grade 3 through 5 events; interestingly, after reduction of gemcitabine, the rate of VTE decreased markedly ( $41 \%$ to $8 \% ; P=.023$ ). Dose reductions for toxicity occurred in most patients $(69.8 \%)$. Complete and partial responses were seen in $8(19 \%)$ and 23 patients (53\%), respectively. Although the overall response rate of $72 \%$ was impressive, the median PFS of 8.2 months did not meet the prespecified study end point. Beyond this phase II effort, the role of bevacizumab in bladder cancer may be better answered by an ongoing phase III study comparing $\mathrm{GC}$ and $\mathrm{GC}$ with bevacizumab in patients with metastatic disease (ClinicalTrials.gov identifier: NCT00941331). Another phase II study led by MD Anderson Cancer Center explored the combination of DD-MVAC with bevacizumab as neoadjuvant therapy for MIBC. ${ }^{87}$ Preliminary results show a pTO rate similar to that for DD-MVAC alone, suggesting a minimal contribution of bevacizumab to overall efficacy.

Downstream of VEGF, mTOR also plays a key role in angiogenesis. ${ }^{88}$ Expression of $\mathrm{mTOR}$ and related mediators (ie, p-4E-BP1) has been shown to have prognostic value, and in preclinical models of bladder cancer, mTOR inhibition seems to have an antitumor effect. ${ }^{89,90}$ In a phase II study, 15 patients with refractory metastatic bladder cancer were treated with weekly temsirolimus, a potent intravenously administered mTOR inhibitor approved for advanced RCC.91,92 The study was stopped because of a lack of observed efficacy; PFS and OS of 2.5 and 3.5 months, respectively, were reported. Despite these discouraging results, a recent study suggests the potential utility of mTOR inhibitors in bladder cancer in the setting of certain mutations. Specifically, loss of function mutations in tuberous sclerosis complex 1 (TSC1) and neurofibromatosis type 2(NF2), upstream of mTOR, resulted in exquisite sensitivity to everolimus in one patient found to have both of these mutations. ${ }^{93}$ Although these mutations are anticipated to occur in a small fraction of patients with bladder cancer, they may serve as important biomarkers. Everolimus is currently being assessed in a phase II study conducted by HOG, in which cisplatin-ineligible patients with metastatic disease are randomized to everolimus with or without paclitaxel (ClinicalTrials.gov identifier: NCT01215136).

Multiple other studies are underway to explore distinct molecular signaling pathways in bladder cancer. Based on data suggesting a key role of fibroblast growth factor receptor (FGFR) aberrations in bladder cancer pathogenesis and therapeutic resistance, ${ }^{94-96}$ the small molecule FGFR inhibitor dovitinib is being assessed in combination with platinum-based chemotherapy for patients with newly diagnosed metastatic disease (ClinicalTrials. gov identifier: NCT01496534). Additionally, given the emerging data suggesting the role of heat-shock protein ( $\mathrm{Hsp}$ ) in conferring chemotherapy resistance, an ongoing randomized phase II study is exploring the combination of GC with OGX-427, an antisense oligonucleotide to Hsp27 (ClinicalTrials. gov identifier: NCT01454089). ${ }^{97}$

\section{Conclusions}

The systemic management of bladder cancer has changed little over time. In the setting of metastatic disease, cisplatin-based chemotherapy remains the cornerstone of treatment for both perioperative and metastatic disease. Despite consensus guidelines supported by prospective trials and meta-analyses stipulating that neoadjuvant chemotherapy be considered for all appropriate patients with muscle-invasive disease, use remains low. With respect to adjuvant therapy, establishing any consensus is challenging given the poor accrual and methodologic flaws present in the prospective studies to date, underscoring the rationale for administering chemotherapy in the preoperative setting rather than after surgery.

As is the case with other cancers, the hope is that the management of bladder cancer will ultimately improve based on a better understanding of its biology. Although the studies assessing targeted therapies described herein have not yielded consistently promising results, perhaps using these drugs in yetto-be-defined subsets of patients who harbor specific molecular alterations will lead to improved efficacy. The search for biologic driver molecular alterations in bladder cancer is well underway. Until these explorations mature, clinicians will be confined to the same cytotoxic agents in the current treatment algo- 
Bladder Cancer: Systemic Therapy

rithms. However, the evidence is clear that even the decades-old tools available to cure locally advanced bladder cancer are underused. As Bajorin and Herr ${ }^{98}$ point out in their thoughtful editorial, based on the slow uptake of consensus guideline recommendations with respect to neoadjuvant chemotherapy, those in the urologic oncology community seem to be the last to appreciate that a paradigm shift has occurred. Despite the clear and consistent outcome of multiple neoadjuvant studies showing a survival benefit and increase in cure fraction with cisplatinbased chemotherapy, most centers have not yet incorporated the practice as standard of care.

\section{References}

1. Pal SK, Williams S, Josephson DY, et al. Novel therapies for metastatic renal cell carcinoma: efforts to expand beyond the VEGF/mTOR signaling paradigm. Mol Cancer Ther 2012;11:526537.

2. Pal SK, Sartor O. Current paradigms and evolving concepts in metastatic castration-resistant prostate cancer. Asian J Androl 2011;13:683-689.

3. Yagoda A, Watson RC, Gonzalez-Vitale JC, et al. Cisdichlorodiammineplatinum(II) in advanced bladder cancer. Cancer Treat Rep 1976;60:917-923.

4. Pal SK, Lewis B, Sartor O. Management of docetaxel failures in metastatic castrate-resistant prostate cancer. Urol Clin North Am 2012;39:583-591.

5. Grossman HB, Natale RB, Tangen CM, et al. Neoadjuvant chemotherapy plus cystectomy compared with cystectomy alone for locally advanced bladder cancer. N Engl J Med 2003;349:859_ 866.

6. Griffiths G, Hall R, Sylvester R, et al. International phase III trial assessing neoadjuvant cisplatin, methotrexate, and vinblastine chemotherapy for muscle-invasive bladder cancer: long-term results of the BA06 30894 trial. J Clin Oncol 2011;29:2171-2177.

7. Clark PE, Agarwal N, Biagioli MC, et al. NCCN Clinical Practice Guidelines in Oncology for Bladder Cancer, version 1.2013. Available at NCCN.org. Accessed January 10, 2013.

8. Neoadjuvant cisplatin,methotrexate, and vinblastine chemotherapy for muscle-invasive bladder cancer: a randomised controlled trial. Lancet 1999;354:533-540. Erratum Lancet 1999;354:1650.

9. Scher HI, Yagoda A, Herr HW, et al. Neoadjuvant M-VAC (methotrexate, vinblastine, doxorubicin and cisplatin) effect on the primary bladder lesion. J Urol 1988;139:470-474.

10. Sternberg CN, de Mulder PH, Schornagel JH, et al. Randomized phase III trial of high-dose-intensity methotrexate, vinblastine, doxorubicin, and cisplatin (MVAC) chemotherapy and recombinant human granulocyte colony-stimulating factor versus classic MVAC in advanced urothelial tract tumors: European Organization for Research and Treatment of Cancer protocol no. 30924. J Clin Oncol 2001;19:2638-2646.

11. Blick C, Hall P, Pwint $T$, et al. Accelerated methotrexate, vinblastine, doxorubicin, and cisplatin (AMVAC) as neoadjuvant chemotherapy for patients with muscle-invasive transitional cell carcinoma of the bladder. Cancer 2012;118:3920-3927.
12. von der Maase H, Sengelov L, Roberts JT, et al. Long-term survival results of a randomized trial comparing gemcitabine plus cisplatin, with methotrexate, vinblastine, doxorubicin, plus cisplatin in patients with bladder cancer. J Clin Oncol 2005;23:4602-4608.

13. Fairey AS, Daneshmand S, Quinn D, et al. Neoadjuvant chemotherapy with gemcitabine/cisplatin vs. methotrexate/ vinblastine/doxorubicin/cisplatin for muscle-invasive urothelial carcinoma of the bladder: a retrospective analysis from the University of Southern California. Urol Oncol, in press.

14. Dash A, Pettus JA IV, Herr HW, et al. A role for neoadjuvant gemcitabine plus cisplatin in muscle-invasive urothelial carcinoma of the bladder: a retrospective experience. Cancer 2008;113:24712477.

15. Scosyrev E, Messing EM, van Wijngaarden E, et al. Neoadjuvant gemcitabine and cisplatin chemotherapy for locally advanced urothelial cancer of the bladder. Cancer 2012;118:72-81.

16. Kaneko G, Kikuchi E, Matsumoto $K$, et al. Neoadjuvant gemcitabine plus cisplatin for muscle-invasive bladder cancer. Jpn J Clin Oncol 2011;41:908-914.

17. Herchenhorn D, Dienstmann R, Peixoto FA, et al. Phase II trial of neoadjuvant gemcitabine and cisplatin in patients with resectable bladder carcinoma. Int Braz J Urol 2007;33:630-638.

18. Weight CJ, Garcia JA, Hansel DE, et al. Lack of pathologic downstaging with neoadjuvant chemotherapy for muscle-invasive urothelial carcinoma of the bladder: a contemporary series. Cancer 2009;115:792-799.

19. Pal SK, Ruel NH, Wilson TG, Yuh BE. Retrospective analysis of clinical outcomes with neoadjuvant cisplatin-based regimens for muscle-invasive bladder cancer. Clin Genitourin Cancer 2012;10:246-250.

20. Yuh BE, Ruel N, Wilson TG, et al. Pooled analysis of clinical outcomes with neoadjuvant cisplatin and gemcitabine chemotherapy for muscle-invasive bladder cancer. J Urol 2013;189:1682-1686.

21. Advanced Bladder Cancer Meta-analysis Collaboration. Neoadjuvant chemotherapy in invasive bladder cancer: update of a systematic review and meta-analysis of individual patient data advanced bladder cancer $(\mathrm{ABC})$ meta-analysis collaboration. Eur Urol 2005;48:202-205; discussion 205-206.

22. Winquist E, Kirchner TS, Segal R, et al. Neoadjuvant chemotherapy for transitional cell carcinoma of the bladder: a systematic review and meta-analysis. J Urol 2004;171:561-569.

23. Fedeli U, Fedewa SA, Ward EM. Treatment of muscle invasive bladder cancer: evidence from the National Cancer Database, 2003 to 2007. J Urol 2011;185:72-78.

24. Donat SM. Integrating perioperative chemotherapy into the treatment of muscle-invasive bladder cancer: strategy versus reality. J Natl Compr Canc Netw 2009; 7:40-47.

25. Paz-Ares LG, Solsona E, Esteban E, et al. Randomized phase III trial comparing adjuvant paclitaxel/gemcitabine/cisplatin (PGC) to observation in patients with resected invasive bladder cancer: results of the Spanish Oncology Genitourinary Group (SOGUG) 99/01 study [abstract]. J Clin Oncol 2010;28(Suppl):Abstract LBA4518.

26. Cognetti F, Ruggeri EM, Felici A, et al. Adjuvant chemotherapy (AC) with cisplatin + gemcitabine (CG) versus chemotherapy (CT) at relapse (CR) in patients (pts) with muscle-invasive bladder cancer (MIBC) submitted to radical cystectomy (RC). An Italian multicenter randomised phase III trial [abstract]. J Clin Oncol 2008;26(Suppl):Abstract 5023.

27. Cognetti F, Ruggeri EM, Felici A, et al. Adjuvant chemotherapy 
Pal et al

with cisplatin and gemcitabine versus chemotherapy at relapse in patients with muscle-invasive bladder cancer submitted to radical cystectomy: an Italian, multicenter, randomized phase III trial. Ann Oncol 2012;23:695-700.

28. Advanced Bladder Cancer Meta-analysis Collaboration. Adjuvant chemotherapy in invasive bladder cancer: a systematic review and meta-analysis of individual patient data Advanced Bladder Cancer (ABC) Meta-analysis Collaboration. Eur Urol 2005;48:189-199, discussion 199-201.

29. Svatek RS, Shariat SF, Lasky RE, et al. The effectiveness of offprotocol adjuvant chemotherapy for patients with urothelial carcinoma of the urinary bladder. Clin Cancer Res 2010;16:44614467.

30. Logothetis CJ, Dexeus FH, Finn L, et al. A prospective randomized trial comparing MVAC and CISCA chemotherapy for patients with metastatic urothelial tumors. J Clin Oncol 1990;8:1050-1055.

31. Sternberg $\mathrm{CN}$, de Mulder $\mathrm{P}$, Schornagel JH, et al. Seven year update of an EORTC phase III trial of high-dose intensity M-VAC chemotherapy and G-CSF versus classic M-VAC in advanced urothelial tract tumours. Eur J Cancer 2006;42:50-54.

32. von der Maase H, Hansen SW, Roberts JT, et al. Gemcitabine and cisplatin versus methotrexate, vinblastine, doxorubicin, and cisplatin in advanced or metastatic bladder cancer: results of a large, randomized, multinational, multicenter, phase III study. J Clin Oncol 2000;18:3068-3077.

33. Lorusso V, Crucitta E, Silvestris N, et al. Randomised, openlabel, phase II trial of paclitaxel, gemcitabine and cisplatin versus gemcitabine and cisplatin as first-line chemotherapy in advanced transitional cell carcinoma of the urothelium. Oncol Rep 2005;13:283-287.

34. Bellmunt J, von der Maase H, Mead GM, et al. Randomized phase III study comparing paclitaxel/cisplatin/gemcitabine and gemcitabine/ cisplatin in patients with locally advanced or metastatic urothelial cancer without prior systemic therapy: EORTC Intergroup study 30987. J Clin Oncol 2012;30:1107-1113.

35. Bellmunt J, Theodore C, Demkov T, et al. Phase III trial of vinflunine plus best supportive care compared with best supportive care alone after a platinum-containing regimen in patients with advanced transitional cell carcinoma of the urothelial tract. J Clin Oncol 2009;27:4454-4461.

36. Mamtani R, Vaughn DJ. Vinflunine in the treatment of advanced bladder cancer. Expert Rev Anticancer Ther 2011;11:13-20.

37. Lorusso V, Pollera CF, Antimi M, et al. A phase II study of gemcitabine in patients with transitional cell carcinoma of the urinary tract previously treated with platinum. Italian Co-operative Group on Bladder Cancer. Eur J Cancer 1998;34:1208-1212.

38. McCaffrey JA, Hilton S, Mazumdar M, et al. Phase II trial of docetaxel in patients with advanced or metastatic transitional-cell carcinoma. J Clin Oncol 1997;15:1853-1857.

39. Papamichael D, Gallagher CJ, Oliver RT, et al. Phase II study of paclitaxel in pretreated patients with locally advanced/metastatic cancer of the bladder and ureter. Br J Cancer 1997;75:606-607.

40. Vaughn DJ, Broome CM, Hussain M, et al. Phase II trial of weekly paclitaxel in patients with previously treated advanced urothelial cancer. J Clin Oncol 2002;20:937-940.

41. Dreicer R, Li H, Cooney MM, et al. Phase 2 trial of pemetrexed disodium and gemcitabine in advanced urothelial cancer (E4802): a trial of the Eastern Cooperative Oncology Group. Cancer 2008;112:2671-2675.

42. Sweeney CJ, Roth BJ, Kabbinavar FF, et al. Phase II study of pemetrexed for second-line treatment of transitional cell cancer of the urothelium. J Clin Oncol 2006;24:3451-3457.

43. von der Maase H, Lehmann J, Gravis G, et al. A phase II trial of pemetrexed plus gemcitabine in locally advanced and/or metastatic transitional cell carcinoma of the urothelium. Ann Oncol 2006;17:1533-1538.

44. Krege S, Rembrink V, Borgermann C, et al. Docetaxel and ifosfamide as second line treatment for patients with advanced or metastatic urothelial cancer after failure of platinum chemotherapy: a phase 2 study. J Urol 2001;165:67-71.

45. Pagliaro LC, Millikan RE, Tu SM, et al. Cisplatin, gemcitabine, and ifosfamide as weekly therapy: a feasibility and phase II study of salvage treatment for advanced transitional-cell carcinoma. J Clin Oncol 2002;20:2965-2970.

46. Bhattacharyya M, Powles T, Mutsvangwa K, et al. A phase II study of mitomycin, fluorouracil, folinic acid, and irinotecan (MFI) for the treatment of transitional cell carcinoma of the bladder. Urol Oncol, in press.

47. Highley MS, Griffiths GO, Uscinska BM, et al. A phase II trial of continuous 5-fluorouracil in recurrent or metastatic transitional cell carcinoma of the urinary tract. Clin Oncol (R Coll Radiol) 2009;21:394-400.

48. Apolo AB, Ostrovnaya I, Halabi S, et al. Prognostic model for predicting survival of patients with metastatic urothelial cancer treated with cisplatin-based chemotherapy. J Natl Cancer Inst 2013;105:499-503.

49. Sonpavde G, Pond GR, Fougeray R, et al. Time from prior chemotherapy enhances prognostic risk grouping in the second-line setting of advanced urothelial carcinoma: a retrospective analysis of pooled, prospective phase 2 trials. Eur Urol 2013;63:717-723.

50. Galsky MD, Chen GJ, Oh WK, et al. Comparative effectiveness of cisplatin-based and carboplatin-based chemotherapy for treatment of advanced urothelial carcinoma. Ann Oncol 2012;23:406-410.

51. Dreicer R, Manola J, Roth BJ, et al. Phase III trial of methotrexate, vinblastine, doxorubicin, and cisplatin versus carboplatin and paclitaxel in patients with advanced carcinoma of the urothelium. Cancer 2004;100:1639-1645.

52. Dogliotti L, Carteni G, Siena S, et al. Gemcitabine plus cisplatin versus gemcitabine plus carboplatin as first-line chemotherapy in advanced transitional cell carcinoma of the urothelium: results of a randomized phase 2 trial. Eur Urol 2007;52:134-141.

53. Petrioli R, Frediani B, Manganelli A, et al. Comparison between a cisplatin-containing regimen and a carboplatin-containing regimen for recurrent or metastatic bladder cancer patients. A randomized phase II study. Cancer 1996;77:344-351.

54. Galsky MD, Hahn NM, Rosenberg J, et al. A consensus definition of patients with metastatic urothelial carcinoma who are unfit for cisplatin-based chemotherapy. Lancet Oncol 2011;12:211-214.

55. Raj GV, Iasonos A, Herr H, Donat SM. Formulas calculating creatinine clearance are inadequate for determining eligibility for cisplatin-based chemotherapy in bladder cancer. J Clin Oncol 2006;24:3095-3100.

56. Carles J, Nogue M, Domenech $M$, et al. Carboplatin-gemcitabine treatment of patients with transitional cell carcinoma of the bladder and impaired renal function. Oncology 2000;59:24-27.

57. Linardou H, Aravantinos G, Efstathiou E, et al. Gemcitabine and carboplatin combination as first-line treatment in elderly patients and those unfit for cisplatin-based chemotherapy with advanced bladder carcinoma: phase II study of the Hellenic Co-operative Oncology Group. Urology 2004;64:479-484.

58. Tsuruta $H$, Inoue $T, N$ arita $S$, et al. Combination therapy consisting of gemcitabine, carboplatin, and docetaxel as an active treatment 
Bladder Cancer: Systemic Therapy

for advanced urothelial carcinoma. Int J Clin Oncol 2011;16:533538.

59. De Santis M, Bellmunt J, Mead G, et al. Randomized phase II/ III trial assessing gemcitabine/carboplatin and methotrexate/ carboplatin/vinblastine in patients with advanced urothelial cancer who are unfit for cisplatin-based chemotherapy: EORTC study 30986. J Clin Oncol 2012;30:191-199.

60. Galsky MD, Iasonos A, Mironov S, et al. Phase II trial of dosedense doxorubicin plus gemcitabine followed by paclitaxel plus carboplatin in patients with advanced urothelial carcinoma and impaired renal function. Cancer 2007;109:549-555

61. Turkolmez K, Beduk Y, Baltaci S, et al. Gemcitabine plus vinorelbine chemotherapy in patients with advanced bladder carcinoma who are medically unsuitable for or who have failed cisplatin-based chemotherapy. Eur Urol 2003;44:682-686.

62. Ricci S, Galli L, Chioni A, et al. Gemcitabine plus epirubicin in patients with advanced urothelial carcinoma who are not eligible for platinum-based regimens. Cancer 2002;95:1444-1450.

63. Carles J, Esteban E, Climent M, et al. Gemcitabine and oxaliplatin combination: a multicenter phase II trial in unfit patients with locally advanced or metastatic urothelial cancer. Ann Oncol 2007;18:1359-1362.

64. Gandour-Edwards R, Lara PN Jr, Folkins AK, et al. Does HER2/ neu expression provide prognostic information in patients with advanced urothelial carcinoma? Cancer 2002;95:1009-1015.

65. Lae M, Couturier J, Oudard S, et al. Assessing HER2 gene amplification as a potential target for therapy in invasive urothelial bladder cancer with a standardized methodology: results in 1005 patients. Ann Oncol 2010;21:815-819.

66. Rink M, Chun FK, Dahlem R, et al. Prognostic role and HER2 expression of circulating tumor cells in peripheral blood of patients prior to radical cystectomy: a prospective study. Eur Urol 2012;61:810-817.

67. Bolenz C, Shariat SF, Karakiewicz PI, et al. Human epidermal growth factor receptor 2 expression status provides independent prognostic information in patients with urothelial carcinoma of the urinary bladder. BJU Int 2010;106:1216-1222.

68. Hussain MH, MacVicar GR, Petrylak DP, et al. Trastuzumab, paclitaxel, carboplatin, and gemcitabine in advanced human epidermal growth factor receptor-2/neu-positive urothelial carcinoma: results of a multicenter phase II National Cancer Institute trial. J Clin Oncol 2007;25:2218-2224.

69. Blehm KN, Spiess PE, Bondaruk JE, et al. Mutations within the kinase domain and truncations of the epidermal growth factor receptor are rare events in bladder cancer: implications for therapy. Clin Cancer Res 2006;12:4671-4677.

70. McConkey DJ, Choi W, Marquis L, et al. Role of epithelial-tomesenchymal transition (EMT) in drug sensitivity and metastasis in bladder cancer. Cancer Metastasis Rev 2009;28:335-344.

71. Petrylak DP, Tangen CM, Van Veldhuizen PJ Jr, et al. Results of the Southwest Oncology Group phase II evaluation (study S0031) of ZD1839 for advanced transitional cell carcinoma of the urothelium. BJU Int 2010;105:317-321.

72. Pruthi RS, Nielsen M, Heathcote S, et al. A phase II trial of neoadjuvant erlotinib in patients with muscle-invasive bladder cancer undergoing radical cystectomy: clinical and pathological results. BJU Int 2010;106:349-354.

73. Wulfing C, Machiels JP, Richel DJ, et al. A single-arm, multicenter, open-label phase 2 study of lapatinib as the second-line treatment of patients with locally advanced or metastatic transitional cell carcinoma. Cancer 2009;115:2881-2890.
74. Grivas P, Agarwal N, Siefker-Radtke AO, et al. Randomized phase II trial of gemcitabine/cisplatin (GC) with or without cetuximab (CET) in patients (pts) with advanced urothelial carcinoma (UC) [abstract]. J Clin Oncol 2012;30(Suppl):Abstract 4506.

75. Wong YN, Litwin S, Vaughn D, et al. Phase II trial of cetuximab with or without paclitaxel in patients with advanced urothelial tract carcinoma. J Clin Oncol 2012;30:3545-3551.

76. Inoue $\mathrm{K}$, Slaton JW, Karashima $\mathrm{T}$, et al. The prognostic value of angiogenesis factor expression for predicting recurrence and metastasis of bladder cancer after neoadjuvant chemotherapy and radical cystectomy. Clin Cancer Res 2000;6:4866-4873.

77. Sonpavde G, Jian W, Liu H, et al. Sunitinib malate is active against human urothelial carcinoma and enhances the activity of cisplatin in a preclinical model. Urol Oncol 2009;27:391-399.

78. Gallagher DJ, Milowsky MI, Gerst SR, et al. Phase II study of sunitinib in patients with metastatic urothelial cancer. J Clin Oncol 2010;28:1373-1379.

79. Galsky MD, Hahn NM, Powles T, et al. Gemcitabine, cisplatin, and sunitinib for metastatic urothelial carcinoma and as preoperative therapy for muscle-invasive bladder cancer. Clin Genitourin Cancer 2013;11:175-181.

80. Necchi A, Mariani L, Zaffaroni N, et al. Pazopanib in advanced and platinum-resistant urothelial cancer: an open-label, single group, phase 2 trial. Lancet Oncol 2012;13:810-816.

81. Pili R, Qin R, Flynn PJ, et al. MC0553: a phase II safety and efficacy study with the VEGF receptor tyrosine kinase inhibitor pazopanib in patients with metastatic urothelial cancer [abstract]. J Clin Oncol 2011;29(Suppl):Abstract 259.

82. Necchi A, Nicolai N, Ortega C, et al. Updated results of INT70/09 phase II study of pazopanib (PZP) monotherapy for patients with relapsed/refractory urothelial cancer (UC) [abstract]. J Clin Oncol 2011;29(Suppl):Abstract 4618.

83. van der Veldt AA, Meijerink MR, van den Eertwegh AJ, et al. Choi response criteria for early prediction of clinical outcome in patients with metastatic renal cell cancer treated with sunitinib. $\mathrm{Br}$ J Cancer 2010;102:803-809.

84. Schmidt $\mathrm{N}$, Hess V, Zumbrunn $\mathrm{T}$, et al. Choi response criteria for prediction of survival in patients with metastatic renal cell carcinoma treated with anti-angiogenic therapies. Eur Radiol 2013;23:632-639.

85. Krajewski KM, Guo M, Van den Abbeele AD, et al. Comparison of four early posttherapy imaging changes (EPTIC; RECIST 1.0, tumor shrinkage, computed tomography tumor density, Choi criteria) in assessing outcome to vascular endothelial growth factortargeted therapy in patients with advanced renal cell carcinoma. Eur Urol 2011;59:856-862.

86. Hahn NM, Stadler WM, Zon RT, et al. Phase II trial of cisplatin, gemcitabine, and bevacizumab as first-line therapy for metastatic urothelial carcinoma: Hoosier Oncology Group GU 04-75. J Clin Oncol 2011;29:1525-1530.

87. Siefker-Radtke AO, Kamat AM, Corn PG, et al. Neoadjuvant chemotherapy with DD-MVAC and bevacizumab in high-risk urothelial cancer: results from a phase II trial at the University of Texas MD Anderson Cancer Center [abstract]. J Clin Oncolo 2012;30(Suppl):Abstract 4523.

88. Pal SK, Figlin RA. Future directions of mammalian target of rapamycin (mTOR) inhibitor therapy in renal cell carcinoma. Target Oncol 2011;6:5-16.

89. Korkolopoulou P, Levidou G, Trigka EA, et al. A comprehensive immunohistochemical and molecular approach to the PI3K/AKT/ mTOR (phosphoinositide 3-kinase/v-akt murine thymoma viral 
Pal et al

oncogene/mammalian target of rapamycin) pathway in bladder urothelial carcinoma. BJU Int 2012;110:E1237-1248.

90. Wu MJ, Chang CH, Chiu YT, et al. Rictor-dependent AKT activation and inhibition of urothelial carcinoma by rapamycin. Urol Oncol 2012;30:69-77.

91. Gerullis $\mathrm{H}$, Eimer $\mathrm{C}$, Ecke $\mathrm{TH}$, et al. A phase II trial of temsirolimus in second-line metastatic urothelial cancer. Med Oncol 2012;29:2870-2876.

92. Hudes G, Carducci M, Tomczak P, et al. Temsirolimus, interferon alfa, or both for advanced renal-cell carcinoma. N Engl J Med 2007;356:2271-2281.

93. Iyer G, Hanrahan AJ, Milowsky MI, et al. Genome sequencing identifies a basis for everolimus sensitivity. Science 2012;338:221.

94. Pandith AA, Shah ZA, Siddiqi MA. Oncogenic role of fibroblast growth factor receptor 3 in tumorigenesis of urinary bladder cancer.
Urol Oncol 2013;31:398-406.

95. Zuiverloon $\mathrm{TC}$, van der $\mathrm{Aa} \mathrm{MN}$, van der Kwast $\mathrm{TH}$, et al. Fibroblast growth factor receptor 3 mutation analysis on voided urine for surveillance of patients with low-grade non-muscleinvasive bladder cancer. Clin Cancer Res 2010;16:3011-3018.

96. Dinney CP, Bielenberg DR, Perrotte P, et al. Inhibition of basic fibroblast growth factor expression, angiogenesis, and growth of human bladder carcinoma in mice by systemic interferon-alpha administration. Cancer Res 1998;58:808-814.

97. Kamada M, So A, Muramaki M, et al. Hsp27 knockdown using nucleotide-based therapies inhibit tumor growth and enhance chemotherapy in human bladder cancer cells. Mol Cancer Ther 2007;6:299-308.

98. Bajorin DF, Herr HW. Kuhn's Paradigms: are those closest to treating bladder cancer the last to appreciate the paradigm shift? J Clin Oncol 2011;29:2135-2137. 